\title{
Soumission chimique : approches pratiques en toxicologie médico-légale
}

\author{
Principles of drug-facilitated \\ crimes investigations
}

\section{Pascal KINTZ, Vincent CIRIMELE, Marion VILLAIN, Antoine TRACQUI, Bertrand LUDES}

\section{$R E ́ S U M E ́$}

De façon cyclique, la presse écrite met en garde ses lecteurs face à l'effet délétère de la consommation de substances psycho-actives, ajoutées à leur insu. Ces produits sont soit des médicaments détournés de leur usage (flunitrazépam, GHB), soit des stupéfiants (cannabis, ecstasy, LSD) ou encore, plus simplement de l'alcool éthylique.

La médecine légale peut'se retrouver impliquée dans la caractérisation de l'usage de ces substances dans le cadre de la soumission chimique.

L'expert en toxicologie peut voir sa tâche rendue difficile par des prélèvements tardifs, des substances à rechercher très faiblement dosées et une interprétation délicate et compliquée, en particulier avec le GHB.

L'objet de ce manuscrit est de proposer une ligne de conduite en matière d'investigations toxicologiques dans les cas de soumission chimique.

\section{MOTS-CLÉS}

Soumission chimique, expertise, toxicologie analytique, prélèvement, cheveux, GHB.

\section{SUMMARY}

The recent increase in reports of drug-facilitated crimes (sexual assault, robbery) has caused alarm in the general public. Drugs involved can be pharmaceuticals, such as benzodiazepines (fiunitrazepam, lorazepam ...), hypnotics (zopiclone, zolpidem), sedatives (for example neuroleptics) or anaesthetics (GHB, ketamine), drugs of abuse, such as cannabis, ecstasy or LSD, or more often ethanol.

To perform successful toxicological examinations, the analyst must follow some important rules: 1 . obtain as soon as possible the corresponding biological specimens (blood, urine and hair), 2. use sophisticated analytical techniques (LC/MS, HS/GC/MS, tandem mass spectrometry); and 3. take care on the interpretation of the findings.

It is the intention of the authors to address a guideline to both medical and analytical personnel for toxicological investigations in case of drug-facilitated crimes.

\section{KEY-WORDS}

sexual assault, robbery, forensic toxicology, hair, GHB. 


\section{Introduction}

La soumission chimique peut être définie comme l'administration de substances psycho-actives à une personne à des fins délictueuses ou criminelles. Dans ce manuscrit, les auteurs feront, à partir de leur expérience judiciaire une synthèse sur les produits utilisés, les prélèvements à effectuer sur les victimes et les aspects analytiques les plus performants.

Les femmes, les enfants et les personnes âgées apparaissent comme les victimes les plus usuelles. L'empoisonnement par un membre de la famille est fréquent chez les enfants (pour obtenir une sédation, soit à visée pédophile, soit simplement pour "avoir la paix") et les personnes âgées (pour détourner leur vigilance), mais la majorité des observations concernent des filles jeunes, à qui leur agresseur administre une substance afin de diminuer leur résistance à l'acte sexuel.

Les produits sont majoritairement incorporés dans des boissons, essentiellement le café ou les préparations alcoolisées. Les aliments, comme les pâtisseries sont peu utilisés.

Très (trop) souvent, il n'y a pas eu de prélèvement biologique effectué lors de la prise en charge et l'expert doit alors travailler sur dossier, ce qui peut être très difficile.

La soumission chimique est un acte criminel dont la prévalence semble être en augmentation, comme le rapporte l'étude de la littérature (1-11).

\section{Quels produits?}

Selon les agresseurs, le produit idéal est celui qui est actif à faible dose (donc aisément incorporable dans une boisson), rapidement soluble en milieu aqueux, sans goût, et dont les effets sont rapides à s'installer, tout en provoquant une amnésie des faits.

Les produits les plus utilisés sont les suivants :

- l'alcool éthylique,

- les benzodiazépines, en pratique quelles soient à demi-vie courte ou longue et notamment le triazolam, le flunitrazépam (mais pas de façon majoritaire), le lorazépam, mais aussi le clonazépam, le bromazépam et enfin le chlorazépate di-potassique,

- les hypnotiques, comme le zolpidem (produit le plus fréquent dans la série de l'Institut de médecine légale de Strasbourg) ou la zopiclone,

- des anesthésiques, comme le GHB ou l'hydrate de chloral, exceptionnellement la kétamine,

- des hallucinogènes tels le LSD, la scopolamine (à partir du Datura), les dérivés de l'ecstasy (MDMA), le cannabis ou les dérivés de la dimethyltryptamine (Ayahuasca),
- des médicaments détournés de leur AMM, comme les neuroleptiques (administrés par des parents à leur enfant, tels l'alimémazine ou la lévomépromazine) ou certains anti-histaminiques $\mathrm{H} 1$ à visée sédative.

L'administration se fait dans la plupart des cas par voie orale dans les boissons, dont le café (probablement du fait de sa couleur et de son amertume naturelle) est le fluide le plus classique.

\section{Quels prélèvements?}

L'interrogatoire d'une victime, reproduit ci-dessous, illustre l'urgence à effectuer les prélèvements biologiques pour documenter au mieux l'observation clinique.

Il s'agit d'une jeune fille de 19 ans, ayant subi une agression sexuelle dans un hôpital et dont l'agresseur était un interne en médecine. Le PV de ses déclarations comporte les phrases suivantes : je ne réalisais plus vraiment ce que je faisais ; ma tête tombait parfois toute seule ; je somnolais, j'avais du mal à lutter contre le sommeil ; j'avais l'impression d'être assommée par la fatigue ; il me parlait, mais je ne me souviens plus de tout ; j'étais comme paralysée et je ne pouvais plus réagir ; cela est venu de manière progressive, environ une demi-heure après avoir bu le café. L'analyse toxicologique révèlera par la suite une administration de zolpidem.

Trois prélèvements doivent être réalisés de façon systématique :

- du sang, prélevé exclusivement sur EDTA (pour éviter la formation in vitro de GHB), en double ( 2 fois $10 \mathrm{ml}$ ). - des urines, sur tube sec (type ECBU), en double (2 fois $30 \mathrm{ml}$ ) dont l'un sera conservé à l'abri de la lumière (pour éviter la dégradation du LSD).

- des cheveux, orientés, en double et coupés au raz du cuir chevelu en occipital, de la taille d'un crayon à papier.

Le sang et les urines sont à conserver au froid, les cheveux sont à maintenir au sec, à température ambiante.

Une feuille de liaison entre le clinicien réalisant la prise en charge médico-légale et le toxicologue est très souhaitable. Cette fiche doit comporter l'heure supposée des faits, le moment des prélèvements, le(s) traitement(s) habituel(s) de la victime (en particulier si elle utilise des hypnotiques le soir) et son éventuel traitement (sédatif, anxiolytique ...) depuis les faits.

La salive et la sueur n'apporte pas des informations complémentaires (2). Dans certains cas, la mise en évidence de marqueurs ou métabolites urinaires permet d'augmenter la fenêtre de détection des xénobiotiques. C'est le cas, par exemple de l'ethyl glucuronide pour 
l'alcool éthylique (2) ou le 2-oxo-3-hydroxy-LSD pour le LSD (11).

Les cheveux constituent l'unique approche satisfaisante pour mettre en évidence une exposition au GHB (12).

Par voie orale, le GHB est facilement absorbé et rapidement éliminé. La caractérisation dans le sang semble impossible après 6 à 8 heures et dans les urines après 8 à 12 heures. Une formation in vitro dans le sang non conservé sur EDTA ou en post mortem, ainsi que des concentrations physiologiques urinaires de l'ordre de quelques $\mathrm{mg} / \mathrm{l}$ peuvent en outre compliquer l'interprétation.

Dans le cadre d'une soumission par GHB, la tâche du toxicologue expert est rendue difficile par plusieurs paramètres : délais souvent importants entre les faits et les prélèvements, demi-vie courte, absence de réponse aux tests immuno-chimiques, analyse non réalisée en routine et concentrations physiologiques de l'ordre de 0,5 à $3 \mathrm{mg} / \mathrm{l}$, même lorsque les prélèvements sont faits dans les conditions optimales.

A l'Institut de Médecine légale de Strasbourg, le protocole qui suit a été mis en place pour documenter un acte de soumission chimique impliquant le GHB.

Il est impératif de prélever les cheveux 1 mois après les faits, afin de suivre, par segmentation, les variations de concentrations.

Les cheveux (une mèche d'au moins 100 cheveux) sont coupés au raz du cuir chevelu, dans des conditions strictes d'alignement, et maintenus par une cordelette. Après décontamination, les cheveux sont coupés en segments de $0,3 \mathrm{~mm}$ sur une longueur de $3 \mathrm{~cm}$. Chaque segment (3-5mg) va ensuite être extrait et purifié, le GHB et GHB- $\mathrm{d}_{6}$ dérivés, puis injectés dans un système de chromatographie gazeuse couplée à un détecteur de masse tandem (Finnigan TSQ 700) en mode impact électronique et SRM. Si on segmente les cheveux d'un même sujet, il est possible d'évaluer un profil d'exposition, en utilisant les concentrations de chaque segment comme référence. En effet, s'il n'y a pas exposition exogène, les concentrations de chaque segment doivent rester très proches. Au contraire, si les segments sont assez courts, par exemple de $0,3 \mathrm{~cm}$, une exposition unique doit conduire à une augmentation ponctuelle de la concentration d'un segment, par rapport aux autres segments qui servent alors de contrôle.

\section{Quelles analyses ?}

L'analyse toxicologique doit être réalisée le plus rapidement possible. Les benzodiazépines sont des produits chimiquement instables, dont la dégradation peut être totale en quelques jours, en particulier pour le flunitrazépam. Il en est de même du LSD. De plus, la formation in vitro du GHB, maintenant bien connue mais mal maîtrisée, est une source d'erreur judiciaire.

Les dépistages par immunochimie doivent être à proscrire. De nombreuses substances ne sont pas reconnues par ces automates, comme le zolpidem, la zopiclone, le GHB, la scopolamine, la kétamine, les neuroleptiques. Dans ces conditions, la règle absolue en matière d'analyse toxicologique pour la caractérisation d'une soumission chimique est la mise en place de techniques séparatives de chromatographie couplées à la spectrométrie de masse.

Compte tenu des molécules à rechercher, telles que définies dans ce texte, l'expert judiciaire se doit de disposer d'un parc analytique impressionnant. En effet, les techniques suivantes doivent être mise en oeuvre :

- chromatographie en phase gazeuse couplée à la spectrométrie de masse (HS/GC/MS) avec introduction par espace de tête, pour les substances volatiles comme l'hydrate de chloral,

- chromatographie en phase gazeuse couplée à la spectrométrie de masse (GC/MS) pour les stupéfiants comme le cannabis, la kétamine, les dérivés de l'ecstasy, le GHB ou les médicaments de type neuroleptiques ou anti-histaminiques,

- chromatographie en phase liquide couplée à la spectrométrie de masse (LC/MS) pour les hypnotiques comme le zolpidem, la zopiclone et les benzodiazépines ou le LSD,

- chromatographie en phase gazeuse couplée à la spectrométrie de masse en tandem (GC/MS/MS/NCI) pour les produits retrouvés à des concentrations particulièrement faibles comme le flunitrazépam, le lorazépam ou le triazolam.

Il s'agit donc de technologies lourdes, qu'il convient de disposer sur un même lieu.

\section{Le cas particulier du GHB}

Dès 1998, et la banalisation de l'usage du GHB aux Etats-Unis dans les affaires de soumission chimique ou de conduite automobile sous influence, la presse scientifique mettait en garde les toxicologues sur une possible formation in vitro (1).

Les seuils de positivité retenus à l'IML de Strasbourg sont donnés dans le tableau 1. Ils tiennent aussi compte de la spécificité post-mortem. Dans ces conditions, un sujet décédé sera déclaré positif au GHB après l'identification simultanée du GHB aux seuils retenus dans le sang, les urines et l'humeur vitrée.

Il apparaît que le GHB est un produit de décomposition 
postmortem, dont la formation semble indépendante du temps et de la température de conservation, mais qui peut être inhibée partiellement par l'ajout de fluorure.

Tableau I : Seuils de positivité du GHB retenus à l'IML de Strasbourg.

\begin{tabular}{|c|c|c|}
\hline Prelèvement & Sujet vivant & Sujet décédé \\
\hline Sang & $5 \mathrm{mg} /$ si prélevé sur EDTA & $50 \mathrm{mg} / /^{*}$ \\
\hline Urines & $10 \mathrm{mg} / \mathrm{l}$ & $10 \mathrm{mg} / \mathrm{l}$ \\
\hline Cheveux & $\begin{array}{c}\text { uniquement par segmentation } \\
\text { quelque soit la couleur }\end{array}$ & $\begin{array}{c}\text { quement par segmentation } \\
\text { quelque soit la couleur }\end{array}$ \\
\hline
\end{tabular}

* avec mise en évidence simultanée du GHB dans les urines et l'humeur vitrée

\section{Quelques exemples}

\section{Exemple 1}

Enfant chimiquement battu : Annaelle, 6 ans, hospitalisée pour incoordination motrice et élocution difficile. Examen clinique sans particularité. Analyse des cheveux : clobazam $=6,28 \mathrm{ng} / \mathrm{mg}$. Faits reconnus par la suite par la maman.

\section{Exemple 2}

Pédophilie : garçon de 14 ans, retrouvé mort au domicile d'un pédophile connu. Autopsie : signes agoniques marqués. Analyse du sang : buprénorphine $(1,1 \mathrm{ng} / \mathrm{ml})$ + norbuprénorphine $(0,2 \mathrm{ng} / \mathrm{ml})+$ nordiazépam $(2,81$ $\mathrm{mg} / \mathrm{l})+$ oxazépam $(0,39 \mathrm{mg} / \mathrm{l})$. Le pédophile déclare n'avoir administré qu'une seule fois ces produits à l'enfant. Analyse des cheveux à l'enfant : buprénorphine $(23 \mathrm{pg} / \mathrm{mg})+$ nordiazépam $(5,23 \mathrm{ng} / \mathrm{mg})+$ oxazépam $(0,08 \mathrm{ng} / \mathrm{mg})$, preuve d'une administration répétée.

\section{Exemple 3}

Viol sous influence : jeune fille de 19 ans, ayant perdu toute notion de la séquence des évènements. Vue aux urgences à $+10 \mathrm{H}$, prise de sang = zolpidem à $39 \mathrm{ng} / \mathrm{ml}$. Analyse des cheveux négative pour le zolpidem (pour éviter d'attribuer la responsabilité à un produit qui ferait partie d'un traitement thérapeutique).

\section{Exemple 4}

Victime dépouillée : homme de 37 ans, abordé dans un parc par une jeune femme qui lui offre un café.

S'endort sur un banc, ne se souvient de plus rien et est délesté de son portefeuille. Vu en consultation à $+11 \mathrm{H}$, prise de sang $=$ lorazépam à $10 \mathrm{ng} / \mathrm{ml}$ et bromazépam à $8 \mathrm{ng} / \mathrm{ml}$

\section{Conclusion}

Chaque cas de soumission chimique (relevant des assises) doit être documenté au mieux, mais le diagnostic toxicologique est difficile. Souvent, les produits utilisés ont des demi-vies courtes, les concentrations circulantes. sont faibles, les prélèvements sont tardifs et les laboratoires d'analyse sont sous-équipés. Il conviendrait, pour ce domaine spécifique, de n'accréditer que quelques laboratoires sur le territoire national.

D'excellentes recommandations pour les analyses toxicologiques ont été publiées par un groupe de travail de l'Académie Américaine des Sciences Judiciaires (AAFS) et constituent une alternative à notre travail (13).

\section{Références}

1. Ghysel M.-H. Le GHB : l'acide gamma hydroxybutyrique. Revue de la littérature. Toxicorama. $1999 ; 11: 1-11$.

2. Kintz P., Villain M., Cirimele V., Goullé J.-P., Ludes B. Usage criminel de substances psycho-actives : le problème de la durée de détection. Acta Clin Belgica. $2002 ; 57$, suppl $1: 24-30$.

3. Wells D. Drug administration and sexual assault : sex in a glass. Sciences \& Justice. $2001 ; 41: 197-199$.

4. Burnat P., Garcia C., Marc B., Allio I., Perrin M., Ceppa F. Agressions sexuelles et soumissions chimiques, un problème d'actualité. Presse Med. 2002 ; 31 : 705-712.

5. Djezzar S., Questel F., Dally S. La soumission médicamenteuse. Courrier des Addictions. $2001 ; 3: 164$.

6. Bismuth C., Dally C., Borron S. Chemical submission : GHB, benzodiazepines and other knock out drops. J Toxicol Clin Toxicol. 1997 ; 35 : 595-598.

7. Djezzar S., Dally S. Utilisation détournée et soumission médicamenteuse : le gamma-OH en question. Med Leg Hosp. $1999 ; 2: 37-39$.

8. Questel F., Bécour B., Dupeyron J.P., Galliot-Guillet M., Diamant-Berger $O$. Soumission médicamenteuse : 4 ans d'expérience aux UMJ de Paris. J Med Leg Droit Med. $2000 ; 43: 459-465$.

9. Ghysel M.-H., Pépin G., Kintz P. La soumission médicamenteuse. Toxicorama. $1998 ; 10: 126-128$.

10. ElSohly M., Lee L., Holzhauer L., Salamone S. Analysis of urine samples in cases of alleged sexual assault. In : Salamone S., ed. Benzodiazepines and GHB. Detection and Pharmacology. Totowa : Humana press. 2001 : 127-144.

11.Pépin G., Gaillard Y. A propos de deux cas d'utilisation originale de la soumission chimique. Ann Toxicol Anal. $2001 ; 13: 122-123$.

12. Kintz P., Cirimele V., Jamey C., Ludes B. Soumission chimique par GHB : cheveux et GC/MS/MS. Ann Toxicol Anal. $2002 ; 14: 129-131$.

13. LeBeau M., Andollo W., Hearn L. et al. Recommendations for toxicological investigations of drug-facilitated sexual assaults. J Forensic Sci. 1999 ; $44: 227-230$. 